\title{
La formation des officiers de l'armée de terre en France, au Royaume-Uni et aux États-Unis. Approche comparative et traductologique
}

\author{
Donatien Lebastard* \\ Aleksandar Stefanovic** \\ Sorbonne Université, Centre de Linguistique en Sorbonne (CeLiSo)
}

Les traductions relatives aux parcours professionnels des officiers dans ces trois pays sont source de difficultés et donc d'erreurs, les glossaires existants n'apportant qu'une aide limitée. L'étude qui se veut une aide à la traduction s'est appuyée sur l'expérience d'officier de réserve des auteurs et sur la documentation disponible sur internet. Selon des modes de recrutement variables car liés au système éducatif du pays, les parcours-types peuvent être les suivants : La formation initiale est de trois ans en France, quatre ans aux États-Unis et un an au Royaume-Uni. La formation intermédiaire préparant au rôle de commandant d'unité tenu pas des capitaines (France et États-Unis) ou commandants (majors au Royaume-Uni) est partout de quelques mois. La formation supérieure s'adressant à des officiers supérieurs (commandant, lieutenant-colonel, colonel) comporte des cycles de quelques mois à une année souvent associés à d'autres enseignements. Le commandement d'un régiment ou d'une brigade suit cette formation. Les droits à retraite sont acquis après 27 à 30 ans de service (France, États-Unis) après un parcours statutaire continu. Au Royaume-Uni, la carrière peut se terminer après un temps de service équivalent, mais peut aussi s'arrêter statutairement plus tôt. Ces considérations ont été synthétisées dans des tableaux récapitulatifs.

Mots-clés : recrutement, écoles, formation, officiers, grades, retraite, France, Royaume-Uni, États-Unis.

Les parcours professionnels des officiers en France, au Royaume-Uni et aux États-Unis présentent des similitudes mais aussi de nombreuses divergences au regard des grades, des périodes de formation, des fonctions occupées à l'issue de ces dernières et des titres universitaires éventuellement accordés. La traduction des appellations définissant les organismes et les cursus n'est pas immédiate

\footnotetext{
*donatien.lebastard@wanadoo.fr

**aleksandar.stefanovic@sorbonne-universite.fr
} 
et peut engendrer des erreurs, les glossaires et/ou dictionnaires français/anglais existants ${ }^{1}$ ne traitant que partiellement ce sujet complexe. Compte tenu de cette lacune paroxystique dans les ouvrages spécialisés, il s'agira donc pour le traducteur d'adopter une véritable stratégie afin de proposer une traduction pertinente, en bref d'adopter une approche traductologique. Il faudra ainsi étudier les différents organismes de formation et les cursus suivis sous les angles suivants : critères de recrutement (âge, niveau d'études préalable pour la formation initiale, grades pour les formations ultérieures) et type de sélection (dossier, entretiens, concours sur épreuves théoriques et sportives), objectifs de la formation, durée de celle-ci, grades et éventuellement diplômes universitaires à la sortie. En parallèle, devront également être mises en exergue les composantes statutaires : engagements réciproques de l'officier et de l'État, contrat de durée limitée ou carrière complète jusqu'à la limite d'âge correspondante. Cette analyse, menée dans le cadre des missions de traduction ou de révision des traductions confiées aux auteurs au profit du $\mathrm{CDEF}^{2}$, permettra en conclusion, dans un souci permanent de parallélisme, de proposer des correspondances traductologiques.

L'étude se concentre sur la carrière d'un officier d'active issu du recrutement direct, ayant choisi l'infanterie et appelé à exercer successivement les fonctions de chef de section, d'adjoint puis de commandant d'unité élémentaire et enfin de chef de corps en alternance avec des passages en état-major. Les différentes étapes, organismes de formation, cycles suivis et fonctions occupées à l'issue sont explicitées pour les trois pays, en soulignant leur positionnement : spécifique à l'arme (ou à la fonction opérationnelle terrestre), interarmes ou interarmées ${ }^{3}$. Compte tenu de la grande diversité des formations possibles, l'analyse se limite aux principales.

\footnotetext{
${ }^{1}$ Voir la bibliographie en fin. Le seul dictionnaire qui analyse plus en détail cette problématique est celui de Jean-Claude Laloire (Laloire 2016) qui propose en effet soit des équivalents britanniques, américains ou canadiens, soit des correspondants traductologiques pour l'appellation des différents centres de formation français. Toutefois les choix de Laloire ne sont pas toujours exempts de critiques notamment en ce qui concerne les équivalents. Ces derniers ne couvrent quasiment jamais les mêmes réalités même s'ils peuvent parfois être proches du correspondant de la langue source ; voir la suite de notre analyse. Une parfaite correspondance n'est donc quasiment jamais possible et cela met constamment le traducteur dans la difficile position de trouver une stratégie à adopter : laisser le centre dans son appellation d'origine avec une éventuelle explicitation entre parenthèses ou en note de bas de page ; trouver l'équivalent le plus proche dans la langue cible avec ou sans explicitation ; ou traduire littéralement.

${ }^{2}$ Centre de doctrine d'emploi des forces (CDEF) situé à l'École Militaire à Paris. Devenu Centre de doctrine et d'enseignement du commandement (CDEC) en 2017. Ce centre est notamment chargé de la traduction de divers productions doctrinales et recueils d'histoire militaire du français vers l'anglais.

${ }^{3}$ La traduction des fonctions opérationnelles peut présenter un problème épineux pour le traducteur. Cf. à ce propos Lebastard \& Stefanovic 2016.
} 
Les considérations faites sont souvent applicables à des carrières d'officiers de carrière d'autres armes ou services, de recrutement semi-direct ou tardif ${ }^{4}$ ainsi que, dans une certaine mesure, aux parcours d'officiers de réserve. Dans le cadre d'accords bilatéraux, les cycles mentionnés sont souvent accessibles à des officiers étrangers au pays.

Compte tenu de la constante évolution de l'enseignement militaire dans les différents pays concernés, la réflexion, qui utilise les informations disponibles à fin 2017, n'a donc pas une valeur immuable.

L'étude est, en outre, accompagnée de diagrammes qui font apparaître, selon le nombre d'années de service, les formations-clés, les grades et les principales fonctions pouvant être occupées. Soulignons que l'étude est essentiellement descriptive et orientée vers la problématique de la traduction du français vers l'anglais, toute considération des mérites respectifs des différents cursus et statuts étant exclue.

Très schématiquement et afin de trouver plus facilement des équivalences, nous structurons les cursus des pays étudiés en trois phases liées aux fonctions (et non aux grades) :

Formation initiale (depuis le recrutement jusqu'à la formation comme chef de section);

Formation intermédiaire (jusqu'à la fonction de commandant d'unité élémentaire) ;

Formation supérieure (au-delà).

\section{Le cursus en France}

Ce cursus, bien connu en France, comporte cependant quelques évolutions récentes qu'il semble nécessaire de mettre en exergue. Une caractéristique importante du système français est le mode de sélection par concours aux principales étapes du parcours. L'autre caractéristique concerne les dispositions statutaires différenciées selon le mode de recrutement. Les officiers issus des écoles de Saint-Cyr Coëtquidan (voir ci-dessous) bénéficient de la possibilité de poursuivre leur carrière jusqu'à la limite d'âge, actuellement à 59 ans pour les officiers des armes, la retraite pouvant, quant à elle, être demandée à 27 ans de service. L'engagement vis-à-vis de l'État est lié à la durée de la formation et est, pour l'École Spéciale Militaire (ESM) de servir cinq ans après la sortie.

\footnotetext{
${ }^{4}$ Concernent les candidats ayant eu un parcours comme sous-officier et éventuellement comme militaire du rang.
} 


\subsection{Formation initiale}

Le campus militaire des Écoles de Saint-Cyr-Coëtquidan (ESCC) abrite deux écoles de formation initiale d'officiers des armes.

L'École Spéciale Militaire de Saint-Cyr-Coëtquidan se caractérise par un recrutement direct de 200 élèves par an. Ce recrutement est majoritairement pourvu par concours ouvert aux candidats issus des classes préparatoires aux grandes écoles (CPGE) ou diplômés du premier cycle de l'enseignement supérieur (Baccalauréat +3 ), âgés de moins de 22 ans au $1^{\text {er }}$ janvier de l'année du concours, avec un taux d'intégration estimé à $5 \%$. Le Recrutement direct sur titres (Officier Sur Titre/OST) concerne environ $10 \%$ de l'effectif d'une promotion et s'adresse aux candidats détenteurs d'un Master ou d'un diplôme équivalent de niveau Bac +5 , âgés de moins de 25 ans. La scolarité est de 3 ans ( 1 an pour les candidats titulaires d'un master recrutés sur titres), les élèves-officiers étant nommés sous-lieutenants (SLT) en début de $3^{\text {e }}$ année. La scolarité débouche sur un titre de Master (diplôme d'ingénieur pour la filière sciences), les OST en étant déjà titulaires. L'objectif est de forger la capacité de commandement de l'officier'.

L'École Militaire Interarmes (EMIA), dont l'objectif est identique à celui de l'ESM, concerne le recrutement semi-direct de 100 élèves par an ; ce recrutement fonctionne, d'une part, sur concours parmi le personnel non-officier, âgé de 23 à 29 ans, titulaires d'un baccalauréat et ayant accompli au moins trois ans de services militaires actifs et, d'autre part, sur dossier suivant les mêmes critères mais visant du personnel avec un premier parcours universitaire (au minimum Bac +2 ). La scolarité se conclut par une licence.

A l'issue de la formation aux ESCC, la division d'application des écoles d'armes forme les officiers destinés à être chefs de section (ou de peloton) au sein des régiments. Pour l'infanterie, d'une durée de 10 mois de septembre à juin, la formation alterne instruction à l'école et sur le terrain que ce soit en France ou à l'étranger (Djibouti, Allemagne...) et se termine par des stages de spécialisation ou qualifiants.

\footnotetext{
5 Aux côtés des 3 bataillons correspondant aux 3 années de l'ESM, le $4^{\text {e }}$ bataillon accueille les stages courts et notamment un stage de 6 mois pour les élèves officiers sous-contrat (OSC) âgés de moins de 32 ans et déjà titulaires d'un diplôme universitaire de niveau Bac+3.
} 


\subsection{Formation intermédiaire}

Celle-ci est dispensée dans deux types d'écoles :

Tout d'abord dans les écoles d'armes, la division d'application forme dans son cycle FFCU (formation des futurs commandants d'unité) les jeunes capitaines ayant vocation à commander une unité élémentaire, c'est-à-dire une compagnie au sein d'un régiment. D'une durée de 14 semaines, la formation alterne cours à l'école et exercices sur le terrain ou en centre de simulation.

L'École d'État-major (EEM) est interarmes et forme les officiers ${ }^{6}$ de l'armée de Terre aux fondamentaux du travail en état-major (EM). Dans ce cadre, l'école organise le stage de 14 semaines délivrant le diplôme d'état-major (DEM) au profit des capitaines de recrutement direct (ESM) et semi-direct (EMIA) ${ }^{7}$.

\subsection{Formation supérieure}

Tout en maintenant pour chaque armée des partenariats avec des écoles et universités civiles, l'Enseignement Militaire Supérieur (EMS), de nature interarmées, dispose de deux organismes de formation propres.

L'École de Guerre (EG), organisme interarmées dénommé dans un passé récent Collège Interarmées de Défense (CID), reçoit chaque année 150 à 200 officiers supérieurs - en majorité des commandants relevant d'une des trois armées ou de la gendarmerie - au terme d'un concours particulièrement sélectif. Les futurs stagiaires ont, dans leur très grande majorité, assumé avec succès au cours d'une première partie de carrière des responsabilités opérationnelles et de commandement au sein de leur armée d'appartenance. Cette école prépare ainsi, pour l'armée de terre, au commandement d'un régiment et au grade de colonel.

Le Centre des Hautes Études Militaires (CHEM) représente le niveau d'enseignement militaire le plus élevé en France. De septembre à juin, les auditeurs, au nombre de 25 après un processus très sélectif suivent une formation de niveau politico-militaire et stratégique. Elle concerne les domaines opérationnels, la préparation du futur, le management et l'organisation des armées ainsi

\footnotetext{
${ }^{6}$ Il existe également un cursus s'adressant aux sous-officiers destinés à servir en état-major.

${ }^{7}$ Ce stage s'adresse également aux officiers sous-contrat/encadrement (OSC E) issus du $4^{\mathrm{e}}$ bataillon de l'ESM. Par ailleurs, l'EEM prépare également le stage de 4 semaines donnant le diplôme d'aptitude aux emplois d'officier supérieur (DAEOS) au profit des capitaines de recrutement tardif. Cf. également à ce propos, supra note 5 .
} 
que les aspects interministériels et internationaux des questions de défense et de sécurité. Les auditeurs participent également aux sessions de l'Institut des Hautes Études de la Défense Nationale (IHEDN) dont le cycle annuel associe également des auditeurs civils.

\subsection{Diagramme résumant le cursus en France}

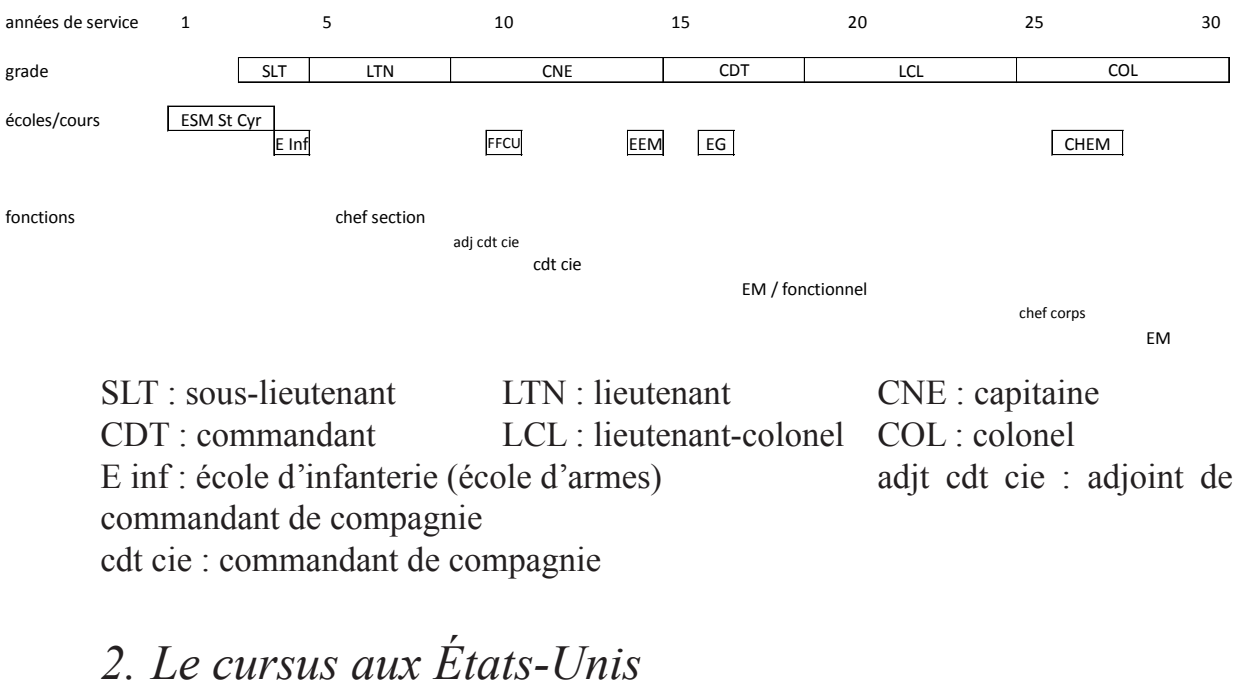

Les formations étudiées sont les principales mentionnées dans le Pamphlet 600-3 (Personnel-General Commissioned Officer Professional Development and Career Management). Les formations dites courtes (inférieures à un mois) ne sont pas prises en considération. Les différentes étapes de formation peuvent se dérouler soit entièrement dans l'armée de terre, soit en partenariat avec échanges de stagiaires avec les autres composantes des armées (US Marine Corps, US Air Force, US Navy) ainsi qu'avec des armées alliées - dont la France. Les éléments résumés dans un diagramme en fin de chapitre reprennent les principaux éléments du Pamphlet 600-3.

Les officiers en activité ont depuis 2005 tous un statut regular (comparable au statut de carrière français) et bénéficient par conséquent d'une stabilité de l'emploi. Les officiers supérieurs peuvent servir pendant 28 ans (lieutenant-colonel) ou 30 ans (colonel) voire au-delà pour les officiers généraux. Par ailleurs, la retraite étant possible dès 20 ans de service, cette solution peut être choisie notamment en cas de non-promotion deux années consécutives. 


\subsection{Formation initiale}

L'United States Military Academy (USMA) à West Point constitue la voie la plus prestigieuse et la plus connue. Le recrutement concerne des candidats âgés de 17 à 23 ans au $1^{\text {er }}$ juillet de l'année d'entrée ayant terminé leur scolarité au lycée (high school) et parfois ayant suivi une année préparatoire optionnelle. La sélection s'effectue d'abord sur dossier : à titre d'illustration, pour 2016, 13827 candidats ont renseigné le questionnaire prenant en compte les résultats scolaires. La recommandation (nomination) d'au moins un élu du congrès (député ou sénateur) est, dans un second temps, requise (4120 candidats l'ont obtenue), l'accent étant ensuite mis sur les épreuves physiques et l'aptitude médicale à l'issue desquelles 2360 candidats ont été sélectionnés. Au final, 1257 cadets sont entrés à l'USMA en première année. Parmi ceux-ci, 100 étaient déjà militaires et ont été recommandés par leur hiérarchie (au minimum par leur commandant d'unité élémentaire). L'objectif est de former en 4 ans des officiers. En fin de scolarité, les cadets sont nommés sous-lieutenants (second lieutenants $2 L t$ ) avec un diplôme de Bachelor of science (licence scientifique quels que soient les enseignements suivis). L'engagement est ensuite de servir pendant cinq ans.

Outre l'USMA, d'autres voies pour devenir officier, non détaillées ici, sont également possibles : le Reserve Officer Training Corps (ROTC) des universités civiles et l'Officer Candidate School (OCS) pour les candidats déjà militaires et sélectionnés (voie représentant la majorité des candidats retenus) ou les civils titulaires d'une licence.

Le Basic Officer Leader Course B (BOLC B) est un cours dispensé dans les écoles d'armes (Branch Schools) ${ }^{8}$ qui s'adresse aux sous-lieutenants nouvellement nommés - sortant de l'USMA ou d'un autre cursus. Il est d'une durée de 12 à 22 semaines selon les armes et spécialités (18 semaines pour l'infanterie). Il complète le BOLC A qui est inclus dans le cursus de l'USMA. Préparant à la fonction de chef de section, il peut être complété par des stages de spécialité (parachutistes, rangers...) ou liés à l'emploi

\footnotetext{
${ }_{8}$ Plusieurs pôles peuvent être mis en exergue. Si certaines écoles sont regroupées dans un même centre d'excellence, notamment pour les armes de mêlée (le Maneuver Center of Excellence ou $M C o E$, rassemble en effet l'Infantry school pour l'infanterie et l'Armor school pour l'arme blindée et cavalerie), d'autres sont dissociées entre plusieurs centres : les armes de l'appui au combat sont ainsi dissociées entre le Maneuver support CoE [qui regroupe le CBRN (Chemical, biological, radiological and nuclear defense - arme nucléaire, radiologique, biologique et chimique - NRBC), l'engineer (génie) et la Military Police (police militaire)] et le Fires (appuis feux) CoE ; enfin certaines écoles sont seules dans leur CoE, comme par exemple l'Army Aviation - Aviation légère de l'Armée de terre (ALAT) ; toutes sont cependant sous l'autorité du Combined Arms Center $(C A C)$ - Centre interarmes et du Training and Doctrine Command (TRADOC) - Commandement de l'entraînement et de la doctrine de l'armée de terre.
} 
de matériels lourds tels que ceux de la gamme Bradley (véhicule blindé chenillé de combat d'infanterie ou de reconnaissance) ou Stryker (véhicule blindé à roues dans ses diverses versions...).

\subsection{Formation intermédiaire}

Le Captains Career Course (CCC) prépare des officiers subalternes au commandement au niveau compagnie et au service des états-majors de niveau régiment/bataillon ou brigade. Il est commun pour les armes de mêlée au centre dédié (Maneuver $\mathrm{CoE})^{9}$ et dure 22 semaines.

\subsection{Formation supérieure}

Le Command and General Staff College (CGSC) (Fort Leavenworth, Kansas) regroupe plusieurs écoles et cours.

Tout d'abord dans la Command and General Staff School (CGSS), le cycle dit ILE Intermediate Level Education ${ }^{10}$ (anciennement Command and General Staff Officer Course) d'une durée de dix mois prépare les commandants, officiers supérieurs à mi-carrière, aux opérations d'envergure dans des environnements interarmées, interministériels et multinationaux. Divers autres cycles ILE sont également possibles dans les organismes partenaires.

Ensuite la School of Advanced Military Studies (SAMS) s'adresse quant à elle à des officiers du grade de commandant ou lieutenant-colonel qui ont terminé leur Intermediate Level Education et qui viennent en majorité du Command and General Staff School. Le cycle Advanced Military Studies Program (AMSP) $)^{11}$ est d'un an et a pour objectif de fournir un enseignement supérieur en sciences militaires aux officiers ayant vocation à commander et à occuper des postes en état-major central. Pour mémoire, la School for Command Preparation (SCP) prépare dans son pre-command course en un mois les lieutenantcolonels et colonels futurs chefs de corps et commandants de brigade à leur commandement.

\footnotetext{
${ }^{9}$ Cf. supra note 8.

${ }^{10}$ Même si l'appellation intermediate est employée, à notre sens, ce cours se situe d'évidence dans la formation supérieure d'officier notamment en regard du cursus français.

11 Les autres programmes (cycles Advanced Strategic Leadership Studies Program-ASLSP, Advanced Strategic Planning and Policy Program-ASP3) peuvent s'étendre sur plusieurs années et s'adressent à un public plus restreint.
} 
Enfin, le plus haut niveau est assuré par les Senior Service Colleges (SSC). Divers établissements relèvent de cette classification, le plus communément suivi est l'Army War College (AWC) (Carlisle Barracks, Pennsylvanie) qui prépare en neuf mois des colonels sélectionnés après leur temps de commandement à des responsabilités au niveau stratégique. Un master en études stratégiques est décerné aux lauréats. Un partenariat existe avec l'IHEDN $^{12}$.

2.4 Diagramme résumant le cursus aux États-Unis

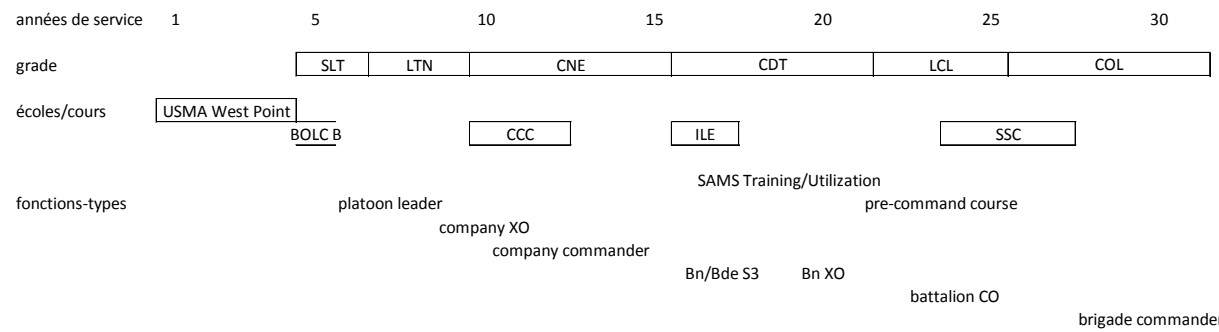

$\mathrm{XO}$ : executive officer (adjoint)

$\mathrm{CO}$ : commanding officer (chef de corps)

S3 : operations officer

$\mathrm{Bn}$ : battalion Bde : brigade

\section{Le cursus au Royaume-Uni}

Si la formation initiale est la même pour tous les officiers, leurs carrières sont structurées par la durée des Commissions (correspondant à des contrats à durée déterminée) qui leur sont accordées et qui correspondent à des étapes de sélection et donc de formation (voir plus loin) pour la promotion au grade supérieur. On distingue en effet : la Short Service Commission (SSC carrière courte de 3 à 8 ans de service après la formation), l'Intermediate Regular Commission (IRC carrière intermédiaire jusqu'à 18 ans de service) et le Regular Commission (RC carrière longue de 35 ans de service ou jusqu'à l'âge de 60 ans, la retraite étant possible à partir de l'âge de 55 ans).

\footnotetext{
${ }^{12}$ Cf. en 1.3.
} 


\subsection{Formation initiale}

Pour la Royal Military Academy Sandhurst (RMAS), le recrutement concerne les candidats de 18 à 29 ans, titulaires au minimum d'un diplôme de fin d'études secondaires ( 2 A levels). En pratique $80 \%$ des cadets sont déjà diplômés d'université ${ }^{13}$. La sélection se fait pour les candidats aptes physiquement sur dossier et entretiens. Le Commissioning Course, cours d'élèves officiers qui est le creuset unique (recrutement interne et externe) est organisé en trois sessions annuelles d'une durée de 44 semaines. A titre d'illustration, en 2014, 540 cadets ont été formés (4280 candidatures ayant été présentées). Le cursus comporte l'attribution de 120 ECTS (European Credits Transfer System) sur 360 requis pour l'obtention d'un Bachelor of arts BA (Hons) (licence en lettres). Il existe également un Late Entry Officer Course (LEOC) de quatre semaines toujours à Sandhurst pour les sous-officiers expérimentés accédant à l'épaulette (grade d'officier dans le jargon militaire).

La formation se poursuit ensuite dans les écoles d'armes. Pour l'infanterie, il s'agit de l'Infantry Battle School (Brecon). Avec des sous-lieutenants (2Lt) sortant de Sandhurst, le Platoon commander's battle course vise à former des chefs de section de combat en 16 semaines.

\subsection{Formation intermédiaire}

Dans un premier temps, le Land Warfare Centre à Warminster propose deux cours :

Le Junior Officers Tactics Awareness Course (JOTAC) prépare en quatre semaines des lieutenants au grade de capitaine. L'objectif final est la connaissance du combat requise pour être adjoint d'une unité élémentaire ou pour occuper une fonction de veille, d'officier de liaison.

Le Captains Warfare Course. Ce cours de huit semaines après une préparation à distance (Military Knowledge 2) est une étape obligatoire pour la promotion au grade de commandant (major).

Ensuite, le Joint Services Command and Staff College (JSCSC) à Shrivenham constitue dans le cadre de la Defence Academy une véritable université

\footnotetext{
${ }^{13}$ Ce système se rapproche en cela de celui du recrutement CPGE de l'ESM (cf. en 1.1). Seul le baccalauréat est requis mais les candidats français ont tous suivi deux à trois ans de préparation de haut niveau.
} 
militaire. Outre des formations supérieures détaillées plus loin, il organise en particulier la formation intermédiaire suivante :

L'Intermediate Command and Staff Course (land) [ICSC(L)] prépare en 30 semaines les jeunes commandants (Army - armée de terre et Royal Marines - infanterie de marine appartenant à la Royal Navy) à des commandements d'unité élémentaire et à des fonctions d'état-major tenues jusqu'au grade de lieutenantcolonel.

\subsection{Formation supérieure}

L'Advanced Command and Staff Course (ACSC) à Shrivenham prépare en 46 semaines des officiers supérieurs des trois armées (commandants et lieutenant-colonels) ${ }^{14}$ sélectionnés pour occuper des fonctions de commandement, en particulier de chef de corps ou de direction. Ce cours est structuré selon les 3 composantes : objectifs, modes d'action et moyens (Ends, Ways, Means). Il peut permettre l'acquisition d'un master.

Le Higher Command and Staff Course (HCSC) à Shrivenham, de création relativement récente (1988), s'adresse à des colonels et généraux de brigade appelés aux plus hautes fonctions de leur armée d'appartenance. En 17 semaines, le cours se focalise sur les problèmes complexes contemporains de nature interarmées et les décisions prises aux niveaux militaro-stratégique et opératif. Sur une promotion de 36 stagiaires, 24 viennent des forces armées britanniques dont 15 de l'armée de terre, les autres stagiaires sont des civils du monde de la défense britannique ou des officiers de nations alliées. A l'issue du cours, le Chartered Management Institute délivre un diplôme de niveau 8 (équivalent à un doctorat).

Le Royal College of Defence Studies (RCDS) à Londres. Cet organisme, de création plus ancienne (1927) est largement ouvert aux étrangers qui constituent les deux-tiers des promotions, celles-ci comptant de 90 à 100 stagiaires. Ainsi 50 nations sontelles représentées dans la promotion 2016. S'adressant en priorité à des colonels anciens et généraux de brigade qui ont démontré leur potentiel pour des responsabilités élevées au sein de leur armée, ce cours de 45 semaines est structuré en trois trimestres dont les thèmes sont respectivement : le contexte stratégique ac-

${ }^{14}$ Ainsi que quelques civils fonctionnaires ou du privé exerçant dans le domaine de la défense. 
tuel et futur, les conflits et la stratégie dans le monde moderne et enfin les problèmes internationaux contemporains. Sous réserve de satisfaire à l'examen de sortie, il délivre un master en sécurité internationale et études stratégiques en partenariat avec le King's College de Londres.

\subsection{Diagramme résumant le cursus au Royaume-Uni}

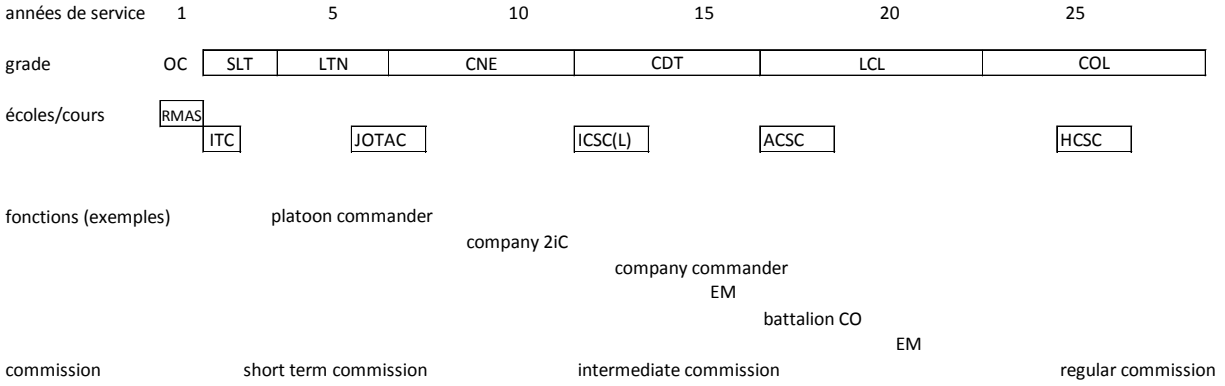

$2 \mathrm{iC}:$ second in command (adjoint)

3.5 Tableaux récapitulatifs des principales formations et propositions de correspondance traductologique français/anglais

\begin{tabular}{|c|c|c|c|}
\hline $\begin{array}{l}\text { Établissement } \\
\text { et durée des } \\
\text { formations }\end{array}$ & France & États-Unis & Royaume-Uni \\
\hline \multirow{4}{*}{ Formation Initiale } & $\begin{array}{c}\text { ESM } 3 \text { ans (1 an } \\
\text { pour les titulaires } \\
\text { d'un master) }\end{array}$ & USMA 4 ans & \multirow{3}{*}{ RMAS 1 an } \\
\hline & Ou EMIA 2 ans & & \\
\hline & & $\begin{array}{c}\text { Ou OCS } 12 \\
\text { semaines } \\
\text { Ou ROTC }\end{array}$ & \\
\hline & $\begin{array}{l}\text { Écoles d'armes } \\
\text { (Infanterie } 10 \\
\text { mois) }\end{array}$ & $\begin{array}{l}\text { Branch schools } \\
\text { (Inf. school } 18 \\
\text { semaines) }\end{array}$ & $\begin{array}{c}\text { Infantry Battle } \\
\text { school } 16 \text { semaines }\end{array}$ \\
\hline
\end{tabular}




\begin{tabular}{|c|c|c|c|}
\hline \multirow{3}{*}{$\begin{array}{l}\text { Formation } \\
\text { Intermédiaire }\end{array}$} & $\begin{array}{l}\text { FFCU École } \\
\text { d'Armes } \\
\text { (Infanterie } 14 \\
\text { semaines) }\end{array}$ & \multirow{3}{*}{ CCC 22 semaines } & JOTAC 4 semaines \\
\hline & EEM 14 semaines & & CWC 8 semaines \\
\hline & & & $\begin{array}{c}\text { ICSC }(\mathrm{L}) 30 \\
\text { semaines }\end{array}$ \\
\hline \multirow{3}{*}{$\begin{array}{l}\text { Formation } \\
\text { supérieure }\end{array}$} & EG 1 an & ILE 1 an & ACSC 46 semaines \\
\hline & CHEM 1 an & ACSC 1 an & HCSC 17 semaines \\
\hline & & SSC 1an & RCDS 45 semaines \\
\hline
\end{tabular}

Le tableau ci-après donne pour chaque entité ou formulation française (liée à la formation) le nom de l'entité comparable ou la formulation équivalente aux États-Unis et au Royaume-Uni.

Pour la traduction proposée, l'expression que nous estimons la plus facilement comprise dans les deux pays a été choisie.

\begin{tabular}{|c|c|c|c|}
\hline France & $\begin{array}{c}\text { Entité } \\
\text { comparable US }\end{array}$ & $\begin{array}{c}\text { Entité comparable } \\
\text { UK }\end{array}$ & $\begin{array}{l}\text { Traduction anglaise } \\
\text { proposée pour le } \\
\text { cursus français }\end{array}$ \\
\hline $\begin{array}{l}\text { Commandement } \\
\text { RH Formation }\end{array}$ & & & $\begin{array}{l}\text { HR \& Education } \\
\text { Command }\end{array}$ \\
\hline $\begin{array}{l}\text { Écoles de Saint- } \\
\text { Cyr Coëtqidan } \\
\text { (ESCC) }\end{array}$ & & \multirow{3}{*}{$\begin{array}{c}\text { Royal Military } \\
\text { Academy } \\
\text { Sandhurst (RMAS) } \\
\text { Commissioning } \\
\text { course }\end{array}$} & $\begin{array}{c}\text { Saint-Cyr Coëtquidan } \\
\text { College }\end{array}$ \\
\hline $\begin{array}{l}\text { ESM Saint-Cyr/ } \\
\text { Coetquidan }\end{array}$ & $\begin{array}{l}\text { US Military } \\
\text { Academy } \\
\text { (USMA) West } \\
\text { Point }\end{array}$ & & $\begin{array}{c}\text { (French) Military } \\
\text { Academy }\end{array}$ \\
\hline EMIA & & & $\begin{array}{l}\text { (French) Combined } \\
\text { Arms Military School }\end{array}$ \\
\hline Élève-officier & Cadet & Officer Cadet & Officer Cadet \\
\hline
\end{tabular}




\begin{tabular}{|c|c|c|c|}
\hline $\begin{array}{l}\text { École du combat } \\
\text { interarmes }\end{array}$ & $\begin{array}{l}\text { Combined } \\
\text { arms center } \\
\text { Maneuver } \\
\text { Centre of } \\
\text { Excellence } \\
\text { (CoE) } \\
\text { Maneuver } \\
\text { Support CoE }\end{array}$ & & $\begin{array}{l}\text { Combined arms } \\
\text { combat school }\end{array}$ \\
\hline $\begin{array}{l}\text { École d'armes } \\
\text { infanterie } \\
\text { artillerie... }\end{array}$ & $\begin{array}{l}\text { Branch schools } \\
\text { : Infantry } \\
\text { School, Armor } \\
\text { school Fires } \\
\text { CoE }\end{array}$ & $\begin{array}{c}\text { Arms schools } \\
\text { Infantry Battle } \\
\text { School }\end{array}$ & $\begin{array}{c}\text { Branch schools } \\
\text { Infantry school } \\
\text { Cavalry/Armor school } \\
\text { Artillery school } \\
\text { Engineer school }\end{array}$ \\
\hline $\begin{array}{l}\text { Cours des chefs de } \\
\text { section }\end{array}$ & BOLC B & $\begin{array}{c}\text { Platoon } \\
\text { commanders battle } \\
\text { course }\end{array}$ & $\begin{array}{c}\text { Platoon leaders/ } \\
\text { commanders course }\end{array}$ \\
\hline $\begin{array}{c}\text { FFCU } \\
\text { formation futurs } \\
\text { commandants } \\
\text { d'unité } \\
\text { élémentaire : } \\
\text { «Cours des } \\
\text { capitaines » }\end{array}$ & $\begin{array}{c}\text { Captains } \\
\text { Career Course } \\
\text { (CCC) }\end{array}$ & $\begin{array}{c}\text { CWC } \\
\mathrm{ICSC}(\mathrm{L})\end{array}$ & $\begin{array}{c}\text { Company } \\
\text { commanders course }\end{array}$ \\
\hline EEM & $\begin{array}{l}\text { Inclus dans } \\
\text { autres cycles }\end{array}$ & $\begin{array}{c}\text { Inclus dans autres } \\
\text { cycles }\end{array}$ & Staff school \\
\hline EMS & & $\begin{array}{c}\text { Senior/ } \\
\text { Executive Officer } \\
\text { Development }\end{array}$ & $\begin{array}{c}\text { Senior Officer } \\
\text { Development } \\
\text { Directorate }\end{array}$ \\
\hline $\begin{array}{c}\text { ESG } \\
\text { (anciennement } \\
\text { CID) }\end{array}$ & $\begin{array}{l}\text { Intermediate } \\
\text { Level } \\
\text { Education ILE } \\
\text { (anciennement } \\
\text { Command \& } \\
\text { general staff } \\
\text { course) } \\
\text { School of } \\
\text { Advanced } \\
\text { Military } \\
\text { Studies SAMS }\end{array}$ & $\begin{array}{c}\text { Advanced } \\
\text { command and staff } \\
\text { course }\end{array}$ & $\begin{array}{l}\text { Advanced command } \\
\text { and staff course }\end{array}$ \\
\hline
\end{tabular}




\begin{tabular}{|c|c|c|c|}
\hline IHEDN & & RCDS & $\begin{array}{l}\text { Defense higher } \\
\text { studies institute }\end{array}$ \\
\hline CHEM & $\begin{array}{c}\text { SSC (Army } \\
\text { War College...) }\end{array}$ & HSCS & $\begin{array}{c}\text { Higher command and } \\
\text { staff course }\end{array}$ \\
\hline Lycée & High school & Secondary school & \\
\hline Baccalauréat (Bac) & $\begin{array}{c}\text { High school } \\
\text { diploma }\end{array}$ & GCSE A levels & \\
\hline $\begin{array}{c}\text { Classes } \\
\text { préparatoires }\end{array}$ & $\begin{array}{l}\text { Preparatory } \\
\text { course }\end{array}$ & & \\
\hline Licence & College degree & Bachelor's degree & Bachelor degree \\
\hline $\begin{array}{c}\text { Diplôme } \\
\text { d'ingénieur }\end{array}$ & $\begin{array}{l}\text { Master's degree } \\
\text { (engineering) }\end{array}$ & & \\
\hline Master & Master degree & Master & Master \\
\hline Doctorat & $\mathrm{Ph} D$ & Doctorate & Doctorate \\
\hline
\end{tabular}

Un certain nombre de pièges se présente et l'attention est attirée sur les points suivants :

Si pour la formation militaire l'ESM, l'USMA et le RMAS sont proches, l'EMIA n'a pas vraiment d'équivalent américain. La formation dispensée à l'OCS est en effet beaucoup plus succincte, trois mois au lieu d'une année de formation militaire. En revanche, il est loisible de comparer l'EMIA à RMAS qui recrute également en semi-direct.

Les stagiaires de l'EG (interarmées) sont essentiellement des commandants comme ceux de l'ILE (US) ou de l'Advanced Command and Staff Course (UK). En revanche, l'US Army War college reçoit des lieutenant-colonels et colonels, ce concept américain restant spécifique à chaque armée (US Army, US Marine Corps, US Air Force, US Navy). Il n'est donc pas souhaitable de traduire l'EG par War College comme c'est très souvent le cas dans des traductions peu scrupuleuses.

En anglais standard, les termes advanced et higher sont proches mais correspondent dans l'enseignement militaire à des niveaux très différents : jeunes commandants dans un cas et colonels dans l'autre. 
Il importe donc de bien distinguer, d'une part, l'établissement, groupe d'écoles (par exemple JSCSC) ou école et, d'autre part, les cycles suivis (ACSC et $\mathrm{HCSC}$ )

L'analyse menée se réfère à la documentation disponible sur internet au moment de la rédaction : sites internet (voir ci-après), documents officiels, glossaires et dictionnaires ${ }^{15}$. Elle est donc sans conteste appelée à évoluer, compte tenu de l'évolution prévisible des formations. Les partenariats et échanges donnant des équivalences pour les officiers suivant des formations à l'étranger constituent un bon moyen de positionner les cycles suivis.

Rappelons enfin que les traductions sont proposées comme un compromis, un équivalent étant rarement possible.

\section{Références bibliographiques}

Boi 2005 : P. Boi, Dictionnaire Interarmées des Termes Militaires et Paramilitaires anglais/français et index français, Paris : La Maison du Dictionnaire.

Goursau 2006 : H. Goursau, Dictionnaire des termes militaires/Dictionary of military terms, anglais/français, Saint-Denis : Éditions Henri Gourseau.

Laloire 2016 : J.-C. Laloire, Dictionnaire des ressources humaines de la défense et de l'entreprise, Paris : L'Harmattan.

Le Lay 2002 : P. Le Lay, Lexique interarmées français/italien/anglais, Paris : La Maison du Dictionnaire.

Lebastard \& Stefanovic 2016 : D. Lebastard et A. Stefanovic, « Fonctions opérationnelles françaises et warfighting functions américaines ; le point de vue du traducteur français/anglais », Lettre du RETEX-Recherche, $\mathrm{n}^{\circ} 28$, Paris : Centre de doctrine d'emploi des forces, $12 \mathrm{p}$.

Pamphlet 600-3 Personnel-General Commissioned Officer Professional Development and Career Management, US Army.

Rostaing 2000 : P. Rostaing, Dictionnaire des Forces terrestres - français/anglais avec index anglais/français, La Maison du Dictionnaire.

\section{Webographie}

Armée de terre française :

www.defense.gouv.fr (ministère de la défense)

www.recrutement.terre.defense.gouv.fr/parcours/eleve-officier-lecole-speciale-militairede-saint-cyr

www.st-cyr.terre.defense.gouv.fr/ (écoles de Saint-Cyr Coëtquidan), www.dems.defense. gouv.fr (Direction de l'enseignement militaire supérieur)

\footnotetext{
${ }^{15}$ Cf. supra note 1.
} 
US Army :

www.usma.edu/SitePages/Home.aspx (US Military Academy West Point) www.apd. army.mil/pdffiles/p600_3.pdf (Pamphlet 600-3)

www.apd.army.mil/pdffiles/r600_8_29.pdf (Army Regulation 600-8-29 Personnel-Officer Promotions)

\title{
UK Army :
}

www.army.mod.uk/training_education/25238.aspx (officers' education)

www.da.mod.uk (Defence Academy of the United Kingdom)

www.army.mod.uk/training_education/24475.aspx (Royal Military Academy Sandhurst).

\author{
Донасијен Лебастар \\ Александар Стефановић
}

\section{Обука за официре копнене војске у Француској, Уједињеном Краљевству и САД-у: Компаративна и традуктолошка метода}

Преводи везани за професионалну каријеру официра у ове три земље представљају извор потешкоћа, али и грешака, нарочито јер постојећи лексикони и речници пружају само ограничену помоћ. Тиме мотивисана, ова студија, има за циљ да помогне у превођењу, те је заснована на искуству аутора (у војној служби) и на документима која су доступна на интернету. Према различитим методама регрутовања у војсци везаним за образовни систем земље, типични курсеви могу бити: Иницијална обука (пандан војној академији у Србији), која има различит рок - у Француској траје три године, четири године у Сједињеним Државама и годину дана у Уједињеном Краљевству. Средња обука којом се припрема за функцију команданта јединице и коју држе капетани (у Француској и Сједињеним Државама) или мајори (у Великој Британији) је свуда неколико месеци. Висока обука за старије официре (мајоре, потпуковнике и пуковнике) укључује циклусе од неколико месеци до годину дана који су често повезани с другим студијама. Команданти бригаде или пука прате ову обуку. Када је реч о вишим официрима, они право на старосну пензију стичу након 27 до 30 година стажа (Француска, Сједињене Државе) и према статусу није могуће да официр буде отпуштен (осим у специфичним ситуацијама). У Уједињеном Краљевству по статусу каријера се може завршити након истог периода службе, али и доста раније. Ова разматрања су приказана у табелама које су део текста.

Кључне речи: регрутовање, школе, обука, официри, оцене, пензија, Француска, Уједињено Краљевство, Сједињене Америчке Државе. 